\title{
UNIFORM DISTRIBUTION WITH RESPECT TO DENSITY
}

\author{
Ligia L. Cristea - Milan PaštéKa
}

\begin{abstract}
The paper deals with a generalisation of uniform distribution. The analogues of Weyl's criterion are derived.
\end{abstract}

\section{Communicated by Oto Strauch}

\section{Introduction}

The notion of uniformly distributed sequence was for the first time defined and studied by Herman Weyl in 1916 in his paper [W]. Since then the theory of uniform distribution was developed in a lot of directions by numerous authors. For a survey we refer to the monographs $[\mathrm{HLA}],[\mathrm{K}-\mathrm{N}],[\mathrm{D}-\mathrm{T}],[\mathrm{S}-\mathrm{P}]$. Since a sequence is a mapping defined on the set of positive integers, the uniform distribution is based on the asymptotic density of coimages of intervals, as defined below.

Let $\mathbb{N}$ be the set of non-negative integers. If for some $A \subset \mathbb{N}$ there exists the limit

$$
\lim _{n \rightarrow \infty} \frac{1}{n}|\{k \leq n: k \in A\}|:=\mathrm{d}(A),
$$

we say that $A$ has asymptotic density, and the value $d(A)$ is called the asymptotic density of $A$. Denote by $\mathcal{D}$ the system that consists of the sets having asymptotic density (see $[\mathrm{PA}]$ ). A sequence $\left\{x_{n}\right\}$ of elements of the interval $[0,1]$ is uniformly distributed modulo 1 if and only if for each subinterval $I \subset[0,1]$ the set $\left\{n ; x_{n} \in I\right\}$ belongs to $\mathcal{D}$ and its asymptotic density is equal to the length of the interval $I$.

2010 Mathematics Subject Classification: 11K06, $11 \mathrm{~B} 05$.

Keywords: uniform distribution, density, Riemann integration.

L. L. Cristea is supported by the Austrian Science Fund (FWF), Project P27050-N26 and by the Austrain-French cooperation project FWF I1136-N26.

M. Paštéka is supported by the grant VEGA 2/0146/14. 
In [KN, Chap. 3] uniform distribution is defined on a compact Hausdorff space $\mathrm{X}$ equipped with a normed Borel measure $P$ and the sequence $\left\{x_{n}\right\}$ in $\mathrm{X}$ is uniformly distributed if and only if

$$
\frac{1}{N} \sum_{n=1}^{N} f\left(x_{n}\right)=\int_{X} f \mathrm{~d} P
$$

for every continuous function $\mathrm{f}$ (called Weyl's Criterion).

In Unsolved Problems, Section 2.1 [UP], the concept of generalisation of uniform distribution is presented as a theory of the integral equation

$$
\int_{X} f(x) \mathrm{d} x=\int_{Y} f(u(y)) \mathrm{d} y,
$$

where $\mathrm{u}(\mathrm{y})$ is called the uniform distribution preserving map, see also [S-P, 1.5].

This paper is inspired by [S-P, 1.5], where a $h$-uniformly distributed sequence $\left\{x_{n}\right\}, x_{n} \in[0 ; 1)$ is defined as follows: for every $x \in[0,1)$ we have

$$
h\left(\left\{n \in \mathbb{N} ; x_{n} \in[0, x)\right\}\right)=x,
$$

where $h$ is a set function defined on a certain class of subsets of $\mathbb{N}$. In this paper we describe uniformity of distribution with respect to a larger class of finitely additive probability measures defined on certain systems of sets.

In Section 2 we introduce the notions of density and $\pi$-uniformly measurable mapping with respect to a density $\pi$. We give some examples of $\pi$-uniformly measurable mappings defined on different spaces, and we prove a necessary and sufficient condition for the existence of a $\pi$-measurable mapping.

In the third section, dedicated to Riemann integrability and the Weyl criterion, we deal with real valued functions defined on a dense subset $\mathbb{M}$ of a compact metric space $\Omega$ equipped with a Borel probability measure. We prove a necessary and sufficient condition for Riemann integrability in terms of uniformly distributed sequences and finally derive a result in terms of asymptotic density.

In Section 4 the role of the set $\mathbb{M}$ from the previous section is played by the set of non-negative integers $\mathbb{N}$. First, we prove results that relate $\pi$-uniform measurability to uniform distribution modulo 1 . In the sequel, the notions and results are extended from real valued mappings to mappings with values in an arbitrary compact metric space endowed with a Borel probability measure. 


\section{UNIFORM DISTRIBUTION WITH RESPECT TO DENSITY}

\section{Preliminaries}

Let $\mathbb{M}$ be an arbitrary set and $\mathcal{Y}$ a system of subsets of $\mathbb{M}$. Then $\mathcal{Y}$ is called a q-algebra of sets if the following three conditions are satisfied:

i) $M \in \mathcal{Y}$,

ii) $A, B \in \mathcal{Y}, A \cap B=\emptyset \Longrightarrow A \cup B \in \mathcal{Y}$,

iii) $A, B \in \mathcal{Y}, A \subset B \Longrightarrow B \backslash A \in \mathcal{Y}$.

It is well known that if in ii) the condition $A \cap B=\emptyset$ is omitted and in iii) the condition $A \subset B$, then $\mathcal{Y}$ is called an algebra of sets.

Let $\pi$ be a finitely additive probability measure on the $q$-algebra $\mathcal{Y}$ fulfilling the condition

$A \in \mathcal{Y}$ if and only if for arbitrary $\varepsilon>0$ there exist $A_{1}, A_{2} \in \mathcal{Y}$ such that

$$
A_{1} \subset A \subset A_{2}, \pi\left(A_{2}\right)-\pi\left(A_{1}\right)<\varepsilon .
$$

We call such a finitely additive probability measure density.

A mapping $x: \mathbb{M} \rightarrow[0,1]$ is called $\pi$-uniformly measurable if for each subinterval $I \subset[0,1]$ the set $x^{-1}(I)$ belongs to $\mathcal{Y}$ and

$$
\pi\left(x^{-1}(I)\right)=|I| \text {. }
$$

The property (11) provides that $x$ is $\pi$-uniformly measurable if and only if (2) holds for a set of intervals $I$, who's set of endpoints is dense in the unit interval.

For a better illustration we show some examples.

ExAMPLE 1. Let $\mathbb{M}=\mathbb{N}$ and $\left\{c_{n}\right\}$ be a sequence of positive real numbers with $\sum_{n=1}^{\infty} c_{n}=\infty$. If for $A \subset \mathbb{N}$ there exists the limit

$$
\lim _{n \rightarrow \infty} \frac{\sum_{k \leq n: k \in A} c_{k}}{\sum_{k=1}^{n} c_{k}}:=d_{c}(A),
$$

then we say that $A$ has weight density with respect to the sequence $\left\{c_{n}\right\}$. In the case $\mathcal{Y}=\mathcal{D}_{c}$, the algebra of the sets having corresponding weight density $\mathrm{d}_{\mathrm{c}}$ (see $[\mathrm{PA}]$ ), and $\pi=\mathrm{d}_{\mathrm{c}}$, the $\pi$-uniformly measurable mapping is a sequence that is uniformly distributed with respect to $d_{C}$.

Example 2. For $\mathbb{M}=\mathbb{N}, \mathcal{D}_{u}$ the algebra of the sets having uniform density $u$ (see $[\mathrm{PA}]$ ), and $\pi=u$, the $\pi$-uniformly measurable mapping is a well distributed sequence.

Example 3. Let $\mathbb{M}=[0, \infty)$. Denote by $\mathcal{Y}$ the family of all Lebesgue measurable sets $S \subset \mathbb{M}$ such that there exists the limit

$$
\pi(S)=\lim _{T \rightarrow \infty} \frac{\lambda(S \cap[0, T])}{T} .
$$


Here the $\pi$-uniformly measurable mapping coincides with the continuous uniform distribution (see $[\mathrm{K}-\mathrm{N}],[\mathrm{D}-\mathrm{T}],[\mathrm{SP}]$ ).

We conclude with an example which is a special case of the object of study in the next section.

Throughout the wohle paper, for any subset $A$ of a topological space, $\operatorname{cl}(A)$ will denote the topological closure of $A$, and $\operatorname{Int}(A)$ its interior in the given space.

EXAMPLE 4. Consider once more $\mathbb{M}=\mathbb{N}$ and $\Omega$ the ring of polyadic integers, (see $[\mathrm{N}],[\mathrm{N} 1])$. Then the set function $\mu^{*}(S)=P(\operatorname{cl}(S))$, where $P$ is the Haar measure on $\Omega$, is called Buck's measure density, (see [BUC], $[\mathrm{PA}]$ ). If $\mathcal{Y}=\mathcal{D}_{\mu}$ is the algebra of all sets measurable in the sense of Buck and $\mu$ the restriction of $\mu^{*}$ on $\mathcal{D}_{\mu}$, we get Buck's uniformly distributed sequences (see [PA], [PA1]). Let us remark that in the paper $[\mathrm{P}-\mathrm{P}]$ more general cases of Borel probability measures on the ring of polyadic integers are constructed, and these can induce densities on $\mathbb{N}$ in an analogous way. Similar examples of density are studied in $[\mathrm{P}-\mathrm{T}]$ in the case when $\mathbb{M}$ is a Dedekind domain with the finite norm property.

The definition of a $\pi$-uniformly measurable mapping leads to the question regarding the existence of such a mapping. We shall characterise it by the following property.

We say that $\pi$ has the Darboux property on $\mathcal{Y}$ if for every $A \in \mathcal{Y}$ and any non negative $\alpha \leq \pi(A)$ there exists $B \subset A, B \in \mathcal{Y}$ with $\pi(B)=\alpha$.

The following result can be proven analogously to Theorem 2.2 in [PS].

Proposition 1. Let $\mathcal{Y}$ be an algebra. The following statements are equivalent:

(1) $\pi$ has the Darboux property on $\mathcal{Y}$.

(2) For every $B \in \mathcal{Y}$ there exists $B_{1} \subset B$ such that $B_{1} \in \mathcal{Y}$ and $\pi\left(B_{1}\right)=$ $\frac{1}{2} \pi(B)$.

(3) For arbitrary $\varepsilon>0$ there exist the sets $C_{1}, \ldots, C_{k}$ such that $\mathbb{N}=C_{1} \cup \cdots \cup$ $C_{k}$ and $\pi\left(C_{j}\right)<\varepsilon, j=1, \ldots, k$.

Theorem 1. Let $\mathcal{Y}$ be an algebra. A $\pi$-uniformly measurable mapping exists if and only if $\pi$ has the Darboux property on $\mathcal{Y}$.

Pr o of. The condition 2 in Proposition 1 provides that there exists a system of decompositions of $\mathbb{M}\left\{U\left(j, 2^{n}\right), j=0, \ldots, 2^{n}-1\right\}, n=1,2, \ldots$, such that $\pi\left(U\left(j, 2^{n}\right)\right)=\frac{1}{2^{n}}$ and $U\left(j, 2^{n}\right)=U\left(2 j, 2^{n+1}\right) \cup U\left(2 j+1,2^{n+1}\right)$.

In the same manner we can consider a system of closed intervals $I\left(j, 2^{n}\right)$ $=\left[\frac{j}{2^{n}}, \frac{j+1}{2^{n}}\right]$, for $j=0, \ldots, 2^{n}-1, n=1,2, \ldots$ For each $k \in \mathbb{N}$ we have a uniquely determined system $U\left(j_{k}^{n}, 2^{n}\right), n=1,2, \ldots$, such that $k \in U\left(j_{k}^{n}, 2^{n}\right)$. The corresponding intervals $I\left(j_{k}, 2^{n}\right)$ form a centered system of closed sets, thus 
its intersection is nonempty. Let us denote by $x(k)$ the element of $\cap_{n=1}^{\infty} I\left(j_{k}^{n}, 2^{n}\right)$. Then we have a mapping $x: \mathbb{M} \rightarrow[0,1]$ such that

$$
k \in U\left(j, 2^{n}\right) \Longrightarrow x(k) \in I\left(j, 2^{n}\right), k \in \mathbb{M} .
$$

Thus $U\left(j, 2^{n}\right) \subset x^{-1}\left(I\left(j, 2^{n}\right)\right)$. Clearly, if $x(k) \in \operatorname{Int}\left(I\left(j, 2^{n}\right)\right)$, then $k \in U\left(j, 2^{n}\right)$ and thus $x^{-1}\left(\operatorname{Int}\left(I\left(j, 2^{n}\right)\right)\right) \subset U\left(j, 2^{n}\right)$, where Int denotes the interior with respect to the topology induced by the Euclidean metric.

Each $y \in[0,1]$ can be contained in some interval $I\left(j, 2^{n}\right) \cup I\left(j+1,2^{n}\right)$. Therefore $x^{-1}(\{y\}) \subset U\left(j, 2^{n}\right) \cup U\left(j+1,2^{n}\right), n \in \mathbb{N}$. This yields $\pi\left(x^{-1}(\{y\})\right)=0$, and thus $\pi\left(x^{-1}\left(\left\{\frac{j}{2^{n}}, \frac{j+1}{2^{n}}\right\}\right)\right)=0$. Considering the inclusions

$$
U\left(j, 2^{n}\right) \backslash x^{-1}\left(\left\{\frac{j}{2^{n}}, \frac{j+1}{2^{n}}\right\}\right) \subset x^{-1}\left(\operatorname{Int}\left(I\left(j, 2^{n}\right)\right)\right) \subset U\left(j, 2^{n}\right),
$$

we have $x^{-1}\left(\operatorname{Int}\left(I\left(j, 2^{n}\right)\right)\right) \in \mathcal{Y}$ and $\pi\left(x^{-1}\left(\operatorname{Int}\left(I\left(j, 2^{n}\right)\right)\right)\right)=2^{-n}$. Since the set of endpoints of intervals $I\left(j, 2^{n}\right)$ is dense in the unit interval we thus have proven that $x$ is $\pi$-uniformly measurable.

The existence of a $\pi$-uniformly measurable mapping implies, by Proposition 1 , that $\pi$ has the Darboux property on $\mathcal{Y}$.

\section{Riemann integrability and the Weyl criterion}

In this section the generalisation of Example 4 will be studied, as announced above. We assume that $\mathbb{M}$ is a dense subset of a compact metric space $(\Omega, \rho)$ equipped with a Borel probability measure $P$.

A sequence $\left\{a_{n}\right\}, a_{n} \in \Omega$ is called uniformly distributed in $\Omega$ if

$$
\lim _{N \rightarrow \infty} \frac{1}{N} \sum_{n=1}^{N} f\left(a_{n}\right)=\int f \mathrm{~d} P,
$$

for every continuous real function $f$ defined on $\Omega$. Equivalently, this means that for every measurable $C \subset \Omega$ with $P(\operatorname{cl}(C) \backslash \operatorname{Int}(C))=0$ the set of indices $\mathbb{A}\left(\left\{a_{n}\right\}, C\right):=\left\{n ; a_{n} \in C\right\}$ has asymptotic density equal to $P(C)$ (see [D-T], $[\mathrm{K}-\mathrm{N}],[\mathrm{S}-\mathrm{P}])$. Here $\operatorname{cl}(C)$ and $\operatorname{Int}(C)$ denote the closure and, respectively, the interior of the set $C$ with respect to the topology on $\Omega$ that is induced by the metric $\rho$.

A measurable set $C \subset \Omega$ with $P(\operatorname{cl}(C) \backslash \operatorname{Int}(C))=0$ is called set of $P$-continuity (see $[\mathrm{D}-\mathrm{T}],[\mathrm{K}-\mathrm{N}],[\mathrm{S}-\mathrm{P}]$ ). 


\section{LIGIA L. CRISTEA — MILAN PAŠTÉKA}

We shall consider the set function

$$
\pi^{*}(S)=P(\operatorname{cl}(S)) .
$$

It can be easily checked that $\pi^{*}$ is a strong submeasure on the system of all subsets of $\mathbb{M}$. This yields that the system of sets $\mathcal{Y}$, consisting of all $A \subset \mathbb{M}$ with $\pi^{*}(A)+\pi^{*}(\mathbb{M} \backslash A)=1$, is an algebra of sets and the restriction $\pi=\pi^{*} \mid \mathcal{Y}$ is a finitely additive probability measure on this algebra, fulfilling the condition (3).

An important role will be played by Riemann integrability and the Riemann integral of bounded real valued functions defined on $\mathbb{M}$.

We rewrite the definition of the Riemann integral from [Bi] and we reprove the criterion from $[\mathrm{Bi}]$ for slightly more general conditions. Then we apply this result in the investigation of uniformly distributed sequences.

Let $C(\Omega)$ be the set of all continuous real functions defined on $\Omega$. For an arbitrary bounded real valued function $f$ defined on $\mathbb{M}$ the values

$$
\int^{*} f=\inf \left\{\int g \mathrm{~d} P ; g \geq f, g \in C(\Omega)\right\}
$$

will be called the upper Riemann integral of $f$ and

$$
\int_{*} f=\sup \left\{\int g \mathrm{~d} P ; g \leq f, g \in C(\Omega)\right\}
$$

the lower Riemann integral of $f$, respectively. The function $f$ will be called Riemann integrable if

$$
\int^{*} f=\int_{*} f:=\int f
$$

in this case this value is called the Riemann integral of $f$.

As usually $B(x, r)$ will be the open ball with center $x \in \Omega$ and radius $r>0$. Since every system of disjoint sets of positive measure is countable we can conclude that for $x \in \Omega$ and positive $r_{1}<r_{2}$ there is $r, r_{1}<r<r_{2}$ such that the ball $B(x, r)$ is a set of $P$-continuity. From the compactness of $\Omega$ we can derive that for each $\alpha$ the set $\Omega$ can be covered by a finite system of open balls with radius smaller than $\alpha$, and from these balls we can construct disjoint sets of $P$-continuity $L_{1}, \ldots, L_{m}$ such that

$$
\Omega=\bigcup_{j=1}^{m} L_{j}
$$

where $\operatorname{diam}\left(\mathrm{L}_{\mathrm{j}}\right)<\alpha$, for $j=1, \ldots, m$. Thus we can construct a system $\mathcal{L}_{n}$, $n=1,2, \ldots$, of disjoint finite covers of $\Omega$ that consist of $P$-continuity sets, $\mathcal{L}_{n}=\left\{L_{1}^{n}, \ldots, L_{m_{n}}^{n}\right\}$ with $\operatorname{diam}\left(\mathrm{L}_{\mathrm{j}}^{\mathrm{n}}\right) \rightarrow 0$ for $n \rightarrow \infty$, uniformly with respect to $j$. 
These can be arranged in such a manner that every set from $\mathcal{L}_{n}$ is a disjoint union of sets from $\mathcal{L}_{n+1}$.

Put $K_{j}^{n}=L_{j}^{n} \cap \mathbb{M}, j=1, \ldots, m_{n}, n=1,2, \ldots$ Denote by $\mathcal{F}$ the set of all real functions of the form $f=\sum_{j=1}^{m_{n}} \beta_{j} \mathcal{X}_{K_{j}^{n}}, \beta_{j} \in \mathbb{R}$. These functions are Riemann integrable and $\int f=\sum_{j=1}^{m_{n}} \beta_{j} P\left(L_{j}^{n}\right)$. By applying Urysohn's Lemma to the sets of $P$-continuity $L_{j}^{n}$ we deduce that in the definition of the upper and lower Riemann integral the set $C(\Omega)$ can be replaced by $\mathcal{F}$.

Thus a bounded function $h$ is Riemann integrable if and only if for arbitrary $\varepsilon>0$ there exist $f_{1}, f_{2} \in \mathcal{F}$ such that $f_{1} \leq h \leq f_{2}$ and $\int\left(f_{2}-f_{1}\right)<\varepsilon$. Clearly, every uniformly continuous real valued function defined on $\mathbb{M}$ is Riemann integrable.

There exists at least one sequence of elements of $\Omega$ that is uniformly distributed in $\Omega$ (see $[H]$, [D-T], $[K-N]$ ). Since $\mathbb{M}$ is dense in $\Omega$ we can assume the existence of at least one uniformly distributed sequence with elements from $\mathbb{M}$.

Denote by $C(\mathbb{M})$ the set of all uniformly continuous real functions defined on this space. This set of functions coincides with the set of all restrictions of continuous real functions defined on $\Omega$ to $\mathbb{M}$. Thus a sequence $\left\{x_{n}\right\}, x_{n} \in \mathbb{M}$, is uniformly distributed if and only if

for each $g \in C(\mathbb{M})$.

$$
\lim _{N \rightarrow \infty} \frac{1}{N} \sum_{n=1}^{N} g\left(x_{n}\right)=\int g,
$$

REMARK 1. Let $R(\mathbb{M})$ be the set of all Riemann integrable real functions defined on $\mathbb{M}$. Clearly, $C(\mathbb{M}) \subset R(\mathbb{M})$ and $\mathcal{F} \subset R(\mathbb{M})$. Moreover,

(i) $R(\mathbb{M})$ is a vector space and $\int$ is a non-negative linear functional on this vector space, and

(ii) $R(\mathbb{M})$ is bounded with respect to the supremum metric and $\int$ is continuous with respect to this metric.

The relationship between uniform distribution and Riemann integrability in the unit interval is described in the paper by de Bruijn and Post [BP]. Later this result was proven in [Bi] by a different method and extended for compact metric spaces. This method was the one exploited in [TW] for uniform distribution. We apply this procedure in our case.

TheOREM 2. Let $f$ be a bounded real valued function defined on $\mathbb{M}$. Then $f$ is Riemann integrable if and only if for every uniformly distributed sequence $\left\{x_{n}\right\}$, $x_{n} \in \mathbb{M}$, there exists the proper limit

$$
\lim _{N \rightarrow \infty} \frac{1}{N} \sum_{n=1}^{N} f\left(x_{n}\right)
$$

in this case this limit is equal to $\int f$. 


\section{LIGIA L. CRISTEA — MILAN PAŠTÉKA}

P r o o f. One implication follows immediately from Weyl's criterion.

Suppose that $f$ is not Riemann integrable. For $x \in \mathbb{M}$, define the functions $H_{n}, h_{n}$, for $n=1,2, \ldots$, as follows:

$$
\begin{gathered}
H_{n}(x)=\sup \left\{f(y) ; y, x \in K_{j}^{n}\right\}, \\
h_{n}(x)=\inf \left\{f(y) ; y, x \in K_{j}^{n}\right\} .
\end{gathered}
$$

Clearly, $H_{n}, h_{n} \in \mathcal{F}$ and $h_{n} \leq f \leq H_{n}$, which implies

$$
\int h_{n} \leq \int_{*} f<\int^{*} f \leq \int_{n}, \quad \text { for } n=1,2, \ldots
$$

Let $\left\{a_{n}\right\}$ be a fixed uniformly distributed sequence of elements of $\mathbb{M}$. Then for every positive integer $s$ we have

$$
\lim _{N \rightarrow \infty} \frac{1}{N} \sum_{n=1}^{N} H_{s}\left(a_{n}\right)=\int H_{s}
$$

This yields that for every $s$ there exists $N(s)$ such that

$$
\frac{1}{N} \sum_{n=1}^{N} H_{s}\left(a_{n}\right)>\int H_{s}-\frac{1}{s}, \quad \text { for } N \geq N(s),
$$

and we can assume that $N(1)<N(2)<\cdots<N(s)<\ldots$ For every positive integer $n$ there exists an uniquely determined $s(n)$ such that $N(s(n)) \leq n<$ $N(s(n)+1)$. For any positive integer $n$, let

$$
H\left(a_{n}\right)=\sup \left\{H_{i}\left(a_{n}\right) ; i>s(n)\right\} .
$$

Then for every $n$ there exists $i(n)>s(n)$ such that $H_{i(n)}\left(a_{n}\right) \geq H\left(a_{n}\right)-\frac{1}{n}$. Suppose that $a_{n} \in K_{j}^{i(n)}$, then we choose $y_{n} \in K_{j}^{i(n)}$ in order that

$$
f\left(y_{n}\right) \geq H_{i(n)}\left(a_{n}\right)-\frac{1}{n} .
$$

From the definition of the sets $K_{j}^{i(n)}$ we see that $\lim _{n \rightarrow \infty} \rho\left(y_{n}, a_{n}\right)=0$, thus the sequence $\left\{y_{n}\right\}$ is uniformly distributed.

Now we can consider

$$
\frac{1}{N} \sum_{n=1}^{N} f\left(y_{n}\right) \geq \frac{1}{N} \sum_{n=1}^{N} H_{i(n)}\left(a_{n}\right)-\frac{1}{N} \sum_{n=1}^{N} \frac{1}{n} .
$$

For $N>N(s(n))$ we have

$$
\frac{1}{N} \sum_{n=1}^{N} f\left(y_{n}\right) \geq \int H_{i(n)}-\frac{1}{i(n)}-\frac{1}{N} \sum_{n=1}^{N} \frac{1}{n} .
$$




\section{UNIFORM DISTRIBUTION WITH RESPECT TO DENSITY}

Since the last two terms tend to 0 , we get

$$
\liminf _{N \rightarrow \infty} \frac{1}{N} \sum_{n=1}^{N} f\left(y_{n}\right) \geq \int^{*} f .
$$

In an analogous way, only considering $h_{n}$ instead of $H_{n}$, we construct a uniformly distributed sequence $\left\{z_{n}\right\}$ with

$$
\limsup _{N \rightarrow \infty} \frac{1}{N} \sum_{n=1}^{N} f\left(z_{n}\right) \leq \int_{*} f .
$$

Clearly, $\lim _{n \rightarrow \infty} \rho\left(y_{n}, z_{n}\right)=0$, therefore every sequence $\left\{u_{n}\right\}$, where $u_{n}=y_{n}$ or $u_{n}=z_{n}$, is uniformly distributed.

Assume that $\left\{M_{k}\right\}$ is a sequence of positive integers with

$$
\lim _{k \rightarrow \infty} \frac{M_{k}}{\sum_{j=1}^{k} M_{j}}=1 .
$$

Define the sequence $\left\{x_{n}\right\}$ as $x_{n}=y_{n}$ for $\sum_{j=1}^{2 k} M_{j} \leq n<\sum_{j=1}^{2 k+1} M_{j}$, and $x_{n}=z_{n}$ for $\sum_{j=1}^{2 k+1} M_{j} \leq n<\sum_{j=1}^{2 k+2} M_{j}$. Then $\left\{x_{n}\right\}$ is uniformly distributed, and

$$
\begin{aligned}
& \liminf _{N \rightarrow \infty} \frac{1}{N} \sum_{n=1}^{N} f\left(x_{n}\right) \leq \int_{*} f, \\
& \limsup _{N \rightarrow \infty} \frac{1}{N} \sum_{n=1}^{N} f\left(x_{n}\right) \geq \int^{*} f .
\end{aligned}
$$

Since $\operatorname{diam}\left(K_{j}^{n}\right) \rightarrow 0$ uniformly for $n \rightarrow \infty$ we get

$$
\operatorname{cl}(S)=\bigcap_{n=1}^{\infty} \bigcup_{S \cap L_{j}^{n} \neq \emptyset} \operatorname{cl}\left(K_{j}^{n}\right) .
$$

Thus, the $P$-continuity of $K_{j}^{n}$ implies $\pi^{*}(S)=\int^{*} \mathcal{X}_{S}$. We get

Corollary 1. A set $S \subset \mathbb{M}$ belongs to $\mathcal{Y}$ if and only if for each uniformly distributed sequence $\left\{x_{n}\right\}$ the set $\mathbb{A}\left(\left\{x_{n}\right\}, S\right)$ belongs to $\mathcal{D}$ and in this case $d\left(\mathbb{A}\left(\left\{x_{n}\right\}, S\right)\right)=\pi(S)$. 
LIGIA L. CRISTEA — MILAN PAŠTÉKA

\section{The set of non-negative integers}

Assume that $\mathbb{M}=\mathbb{N}$ is the set of non-negative integers. Since $\mathbb{N} \subset \Omega$, we can consider sequences of positive integers uniformly distributed in the compact space $(\Omega, \rho, P)$.

A lot of examples of compact spaces containing the set $\mathbb{N}$ as a dense subset are described in the papers $[\mathrm{IPT}],[\mathrm{N}],[\mathrm{N} 1],[\mathrm{P}-\mathrm{P}]$. In this case the mappings $x: \mathbb{N} \rightarrow[0,1]$ are sequences.

The above corollary yields that a mapping $x: \mathbb{N} \rightarrow[0,1]$ is $\pi$-uniformly measurable if and only if for each sequence of positive integers $\left\{k_{n}\right\}$ that is uniformly distributed in $\Omega$,

$$
\lim _{N \rightarrow \infty} \frac{1}{N} \sum_{n=1}^{N} \mathcal{X}_{I}\left(x\left(k_{n}\right)\right)=|I|,
$$

for any arbitrary subinterval $I \subset[0,1)$. Thus by the standard procedure we can prove:

Theorem 3. A sequence $\{x(n)\}$ of elements of the unit interval is $\pi$-uniformly measurable if and only if for each sequence of positive integers $\left\{k_{n}\right\}$ that is uniformly distributed in $\Omega$ we have

$$
\lim _{N \rightarrow \infty} \frac{1}{N} \sum_{n=1}^{N} f\left(x\left(k_{n}\right)\right)=\int_{0}^{1} f(t) \mathrm{d} t,
$$

for every Riemann integrable, (continuous,) real function $f$ defined on $[0,1]$.

By the standard procedure we can derive the following:

Proposition 2. Let $\omega: \Omega \rightarrow[0,1]$ be a continuous function. Suppose that $\{n\}$ is uniformly distributed in $\Omega$. Then the sequence $\{\omega(n)\}$ is $\pi$-uniformly measurable if and only if it is uniformly distributed modulo 1.

If we apply the proof of the main result in [TW] we obtain the part 1 of following assertion. The part 2 follows immediately from Theorem 4

Proposition 3. Let $\omega: \Omega \rightarrow[0,1]$ be a continuous function such that

$$
\int \omega \circ f=\int_{0}^{1} f(t) \mathrm{d} t
$$

(where $\omega \circ f=f(\omega(\cdot))$ is the composition of mappings) for every real continuous function $f$ defined on $[0,1]$. Then 
(1) For every sequence of positive integers $\left\{k_{n}\right\}$ that is uniformly distributed in $\Omega$ the sequence $\left\{\omega\left(k_{n}\right)\right\}$ is uniformly distributed modulo 1 .

(2) If $\{n\}$ is uniformly distributed in $\Omega$, then $\{\omega(n)\}$ is $\pi$-uniformly measurable.

Analogously to uniform distribution, the notion of $\pi$-uniform measurability can be easily extended from the unit interval to an arbitrary compact metric space. Let $\mathcal{M}$ be a compact metric space with Borel probability measure $\tilde{P}$. A mapping $y: \mathbb{N} \rightarrow \mathcal{M}$ is called $\pi$-uniformly measurable if for every set of $\tilde{P}$-continuity $A \subset \mathcal{M}$ we have $y^{-1}(A) \in \mathcal{Y}$ and $\pi\left(y^{-1}(A)\right)=\tilde{P}(A)$.

Theorem 2 yields by the standard procedure, using Urysohn's Lemma, Weyl's criterion for this case:

Proposition 4. A mapping $x: \mathbb{N} \rightarrow \mathcal{M}$ is $\pi$-uniformly measurable if and only if

$$
\lim _{N \rightarrow \infty} \frac{1}{N} \sum_{n=1}^{N} f\left(x\left(k_{n}\right)\right)=\int f \mathrm{~d} \tilde{P}
$$

for every continuous real function defined on $\mathcal{M}$ and every sequence of positive integers $\left\{k_{n}\right\}$ that is uniformly distributed in $\Omega$.

Now we can show the existence property by applying the procedure from $[\mathrm{H}]$. We start by proving the following proposition.

Proposition 5. Let $\pi$ be a density on the set of positive integers defined by (1), having the Darboux property. If $y: \mathbb{N} \rightarrow[0,1]$ is a $\pi$-uniformly measurable mapping and $x: \mathbb{N} \rightarrow[0,1]$ is a mapping such that $\lim _{n \rightarrow \infty}|y(n)-x(n)|=0$, then also $x$ is a $\pi$-uniformly measurable mapping.

Proof. The Darboux property of $\pi$ implies that $\pi(F)=0$ for each finite set $F \subset \mathbb{N}$. Thus for every sequence of positive integers $\left\{k_{n}\right\}$ we have $\lim _{N \rightarrow \infty} \sum_{n \leq N, k_{n} \in F} 1=0$. If $f$ is a continuous real function defined on $[0,1]$, it is also uniformly continuous. Thus for arbitrary $\varepsilon$ there exists $n_{0}$ such that for $n>n_{0}$ we have $|f(x(n))-f(y(n))|<\varepsilon$. This yields that for each sequence of positive integers $\left\{k_{n}\right\}$ that is uniformly distributed in $\Omega$ we have

$$
\limsup _{N \rightarrow \infty} \frac{1}{N} \sum_{n=1}^{N}\left|f\left(x\left(k_{n}\right)\right)-f\left(y\left(k_{n}\right)\right)\right| \leq \varepsilon,
$$

and the assertion follows.

Theorem 4. Assume that $\pi$ is a density on $\mathbb{N}$ defined by (1). If $\pi$ has the Darboux property on $\mathcal{Y}$, then for every compact metric space $\mathcal{M}$ with Borel probability measure $\tilde{P}$ there exists a $\pi$-uniformly measurable mapping $x: \mathbb{N} \rightarrow \mathcal{M}$. 


\section{LIGIA L. CRISTEA — MILAN PAŠTÉKA}

Proof. The compactness of $\mathcal{M}$ provides that there exists a system $\mathcal{S}_{m}=$ $\left\{S_{m}^{j}, j=1, \ldots, r(m)\right\}, S_{m}^{j} \neq \emptyset, m=1,2, \ldots$, of finite closed covers of $\mathcal{M}$, having the following properties:

i) If $n\left(\mathcal{S}_{m}\right)=\max \left\{\operatorname{diam}\left(S_{m}^{j}\right), j=1, \ldots, r(m)\right\}, m=1,2, \ldots$, then

$$
\lim _{m \rightarrow \infty} n\left(\mathcal{S}_{m}\right)=0 .
$$

ii) For every $m$ and $i \neq j, \tilde{P}\left(S_{m}^{i} \cap S_{m}^{j}\right)=0$.

iii) If $m \leq n$, then for every $i \leq r(n)$ there exists a uniquely determined

$$
j=j(i) \leq r(m)
$$

such that $S_{n}^{i} \subset S_{m}^{j}$

iv) The indices in iii) are ordered in such a way that

$$
i_{1}<i_{2} \Rightarrow j\left(i_{1}\right) \leq j\left(i_{2}\right) .
$$

The property i) provides that

v) every real valued continuous function defined on $\mathcal{M}$ can be uniformly approximated by the linear combination of the functions $\mathcal{X}_{S_{m}^{j}}, j=1, \ldots, r(m)$, for suitable $m$.

Moreover, for every $x \in \mathcal{M}$ and any open ball $B$ containing $x$ there exists a suitable $S_{m}^{j}$ such that $x \in S_{m}^{j} \subset B$. Thus

vi) every open set is a union of a countable system of sets $S_{m}^{j}$.

To each cover $\mathcal{S}_{m}$ we can associate the finite system of intervals $I_{m}^{j}$, for $j=1, \ldots, r(m), I_{m}^{j}=\left[\sum_{l=1}^{j-1} \tilde{P}\left(S_{m}^{l}\right), \sum_{l=1}^{j} \tilde{P}\left(S_{m}^{l}\right)\right]$, and thus $\left|I_{m}^{j}\right|=\tilde{P}\left(S_{m}^{l}\right)$, and the endpoints of the intervals $I_{m}^{j}, j=1, \ldots, r(m)$, form a division of the interval $[0,1]$.

Let $x: \mathbb{N} \rightarrow[0,1]$ be a $\pi$-uniformly measurable mapping, its existence being provided by Theorem 1 . The complement of the set of endpoints of the considered intervals is dense in the unit interval. Proposition 5 guarantees that for each dense subset of the unit interval a $\pi$-uniformly measurable mapping can be constructed such that values of this mapping belong to this set. Thus we can assume that the endpoints of $I_{m}^{k}, k=1, \ldots, r(m)$, do not belong to the set $x(M)$.

Each number $\alpha \in[0,1]$ which does not coincide with the endpoints of the intervals $I_{m}^{k}, k=1, \ldots, r(m)$, belongs to exactly one interval $I_{m}^{l(m)}$, for $m=$ $1,2, \ldots$, where $l(m):=l(m, \alpha)$ depends on $\alpha$. Since for every $m=1,2, \ldots$ we have $S_{m+1}^{l(m+1)} \subset S_{m}^{l(m)}$, the compactness of $\mathcal{M}$ guarantees that the set

$$
D(\alpha)=\cap_{m=1}^{\infty} S_{m}^{l(m)}
$$

is not empty. Consider now a mapping $y: \mathbb{N} \rightarrow \mathcal{M}$ such that $y(n) \in D(x(n)), n=$ $1,2, \ldots$ 


\section{UNIFORM DISTRIBUTION WITH RESPECT TO DENSITY}

We prove that this is a $\pi$-uniformly measurable mapping. Let $\left\{k_{n}\right\}$ be an arbitrary sequence of positive integers that is uniformly distributed in $\Omega$. Denote, for $H \subset \mathcal{M}$,

$$
P^{*}(H)=\limsup _{N \rightarrow \infty} \sum_{n=1}^{N} \mathcal{X}_{H}\left(x\left(k_{n}\right)\right),
$$

and

$$
P_{*}(H)=\liminf _{N \rightarrow \infty} \sum_{n=1}^{N} \mathcal{X}_{H}\left(x\left(k_{n}\right)\right) .
$$

Let $\mathcal{Z}$ denote the system of all sets of the form $\cup_{t=1}^{T} S_{m}^{i_{t}}$. Clearly,

$$
\mathcal{X}_{\cup_{t=1}^{T} S_{m}^{i_{t}}}(y(n)) \geq \mathcal{X}_{\cup_{t=1}^{T} I_{m}^{i_{t}}}(x(n)) .
$$

Thus, using the fact that the sequence $\left\{x\left(k_{n}\right)\right\}$ is uniformly distributed in $[0,1]$, we obtain that for every $S \in \mathcal{Z}$ we have $P_{*}(S) \geq \tilde{P}(S)$.

On the other hand, vi) implies that for every open set $G$ we have

$$
\tilde{P}(G)=\sup \{\tilde{P}(S) ; S \subset G, S \in \mathcal{Z}\} .
$$

Thus for every closed set $F$ and every $\varepsilon>0$ there exists a set $S^{\prime} \in \mathcal{Z}$ such that $S^{\prime} \subset \mathcal{M} \backslash F$ and $P\left(S^{\prime}\right)>\tilde{P}(\mathcal{M} \backslash F)-\varepsilon$. Herefrom we obtain

$$
P^{*}(F) \leq 1-P_{*}\left(S^{\prime}\right) \leq 1-\tilde{P}\left(S^{\prime}\right)<1-\tilde{P}(\mathcal{M} \backslash F)+\varepsilon=\tilde{P}(F)+\varepsilon .
$$

Since $\varepsilon$ is arbitrary, we obtain $P^{*}(F) \leq \tilde{P}(F)$. Therefore, for every $S \in \mathcal{Z}$, $P_{*}(S)=P^{*}(S)=\tilde{P}(S)$ holds and the assertion follows from $\left.\mathrm{v}\right)$.

In the following we give examples that illustrate the situation in Theorem 4

ExAmple 5. With the notations in Theorem 4, let $\mathcal{M}=[0,1]$, and $\pi$ be a density on $\mathbb{N}$ that has the Darboux property. We consider $[0,1]$ to be endowed with the measure $\tilde{P}=\mu_{r}$, where $\mu_{r}$ is the binary measure of parameter $r$ on the unit interval, defined below. Thus in this case the equation (2) that defines $\pi$-uniform measurability becomes $\pi\left(x^{-1}(I)\right)=\mu_{r}(I)$.

Consider an arbitrarily fixed real number $r \in(0,1)$, and let $I_{0,0}=[0,1]$, $I_{n, j}=\left[\frac{j}{2^{n}}, \frac{j+1}{2^{n}}\right)$, for $j=1,2, \ldots 2^{n}-2$, and $I_{n, 2^{n}-1}=\left[\frac{2^{n}-1}{2^{n}}, 1\right]$. Then the binomial measure of parameter $r$ on $[0,1]$ is a probability measure that is defined by the conditions

$$
\mu_{r}\left(I_{n+1,2 j}\right)=r \mu_{r}\left(I_{n, j}\right) \quad \text { and } \quad \mu_{r}\left(I_{n+1,2 j+1}\right)=(1-r) \mu_{r}\left(I_{n, j}\right),
$$

for

$$
n=0,1, \ldots, \text { and } j=0,1, \ldots, 2^{n}-1 .
$$

For more details regarding the binomial distribution we refer, e.g., to OSS1, C-P. 
It is easy to see that

$$
\mu_{r}\left(I_{j, 2^{n}}\right)=r^{n-k}(1-r)^{k},
$$

where $k$ is the number of digits 1 in the binary digital expansion of the number $j$. Moreover, $\mu_{r}(\{y\})=0$, for all $y \in[0,1]$.

Now we follow the steps and ideas from the proof of Theorem 11. Under the assumption that $\pi$ has the Darboux property, we have that for each $B \in \mathcal{Y}$ there exists a set

$$
B_{1} \in \mathcal{Y}, \quad B_{1} \subset B \quad \text { such that } \pi\left(B_{1}\right)=r \cdot \pi(B) .
$$

We proceed as in the mentioned proof and obtain, for $n=1,2, \ldots$, the decomposition consisting of the sets $U\left(j, 2^{n}\right)$, for $j=0,1, \ldots, 2^{n}-1$, with $\pi\left(U\left(j, 2^{n}\right)\right)=$ $r^{n-k}(1-r)^{k}$, where $k$ is the number of digits 1 in the binary digital expansion of the number $j$.

REMARK 2. The construction in Example 5 can also be extended analogously, following the above ideas, to the case when $\tilde{P}=\mu_{q, \mathbf{r}}$, where $\mu_{q, \mathbf{r}}$ is the multinomial measure of parameter vector $\mathbf{r}=\left(r_{0}, r_{1}, \ldots, r_{q-1}\right), 0<r_{i}<1, \sum_{i=1}^{q-1} r_{i}=1$, where the role the of the numeration base 2 from Example 5 is here played by the positive integer $q \geq 2$. For more details regarding this measure we refer, e.g., to OSS2, C-P. Moreover, passing from the $n$-th decomposition of $\mathbb{N}$ to the $(n+1)$ th decomposition is given by the relation

$U\left(j, q^{n}\right)=\cup_{k=0}^{q-1} U\left(q j+k, q^{n+1}\right), \quad$ for $\quad j=0,1, \ldots, q^{n}-1, \quad$ where $n=1,2 \ldots$

EXAMPLE 6. With the notations in Theorem 4, let $\mathcal{M}$ be the well-known "two thirds" Cantor set $\mathcal{C}$, and $\pi$ be a density on $\mathbb{N}$ that has the Darboux property. We consider $\mathcal{C}$ to be endowed with the measure $\tilde{P}=\mu_{\mathcal{C}}$, where $\mu_{\mathcal{C}}$ is a probability measure on the Cantor set, defined inductively as follows. Let us start with the set $C_{0}=[0,1]$ and let $\mu_{\mathcal{C}}\left(C_{0}\right)=1$. Let $C_{1}=\left[0, \frac{1}{3}\right] \cup\left[\frac{2}{3}, 1\right]$ and define $\mu_{\mathcal{C}}\left(\left[0, \frac{1}{3}\right]\right)=\mu_{\mathcal{C}}\left(\left[\frac{2}{3}, 0\right]\right)=\frac{1}{2}$. We proceed inductively and obtain at step $k \geq 1$ the set $C_{k}$ as the union of $2^{k}$ closed intervals of length $3^{-k}$ and each of them having the measure $\mu_{\mathcal{C}}$ equal to $2^{-k}$. Thus we obtain, in the limit, a measure whose support is the Cantor set.

This measure coincides with the normalised Hausdorff measure on the Cantor set. For details regarding this measure, see, e.g., [F], [C-T]. We consider the compact metric space $\mathcal{C}$ endowed with the Euclidean metric and the topology induced by the Euclidian topology of the real line. Then any set of the form $I \cap \mathcal{C}$, where $I \subset[0,1]$ is an interval, is a set of $\mu_{\mathcal{C}}$-continuity.

In this case the definition of $\pi$-uniform measurability that we mentioned on the page 10 becomes $\pi\left(x^{-1}(A)\right)=\mu_{\mathcal{C}}(A)$, for every set A of $\mu_{\mathcal{C}}$-continuity. 


\title{
UNIFORM DISTRIBUTION WITH RESPECT TO DENSITY
}

\author{
REFERENCES
}

[Bi] BINDER, C.: Über einen Satz von de Bruijn und Post, Österr. Akad. Wiss. Math.-Naturw. Kl. S B. II, 179 (1971), 233-251.

[B-P] DE BRUIJN, N.G.-POST, K. A.: A remark on uniformly distributed sequences and Riemann integrability Indag. Math. 30 (1968), 149-150.

[BUC] BUCK, R.C.: The measure theoretic approach to density, Amer. J. Math 68 (1946), 560-580.

[C-P] CRISTEA, L. L.-PRODINGER, H.: Moments of distributions related to digital expansions, J. Math. Anal. Appl. 315 (2006), 606-625.

[C-T] CRISTEA, L. L.-TICHY, R. F.: Discrepancies of point sequences on the Sierpinski carpet, Math. Slovaca, 53 (2003), no. 4, 351-367.

[D-T] DRMOTA, M.-TICHY, R.F.: Sequences, Discrepancies and Applications. Springer-Verlag, Berlin Heidelberg, 1997.

[F] FALCONER, K. J.: Fractal Geometry. Mathematical Foundations and Applications. John Wiley \& Sons, Chichester, 1990.

[H] HEDRLIN, Z.: On integration in compact metric spaces, Comment. Math. Univ. Carolinae, 24 (1961), no. 4, 17-19.

[HLA] HLAWKA, E.: Theorie der Gleichverteilung. Bibliographisches Institut, Mannheim, 1979.

[IPT] IACO, M.R.-PAŠTÉKA, M.- TICHY, R.F.: Measure density for set decompositions and uniform distribution, Rend. Circ. Math. Palermo 2 (2015), 323-339.

[K-N] KUIPERS, L.-NIEDERREITER, H.: Uniform Distribution of Sequences. John Wiley and Sons, N.Y. London, Sydney, Toronto, 1974.

[N] NOVOSELOV, E. V.: Topological theory of polyadic numbers, Trudy Tbilis. Mat. Inst. 27 (1960), 61-69. (In Russian)

[N1] New method in the probability number theory, Doklady Akad. Nnauk. Ser. Matem. 28 (1964), no. 2, 307-364. (In Russian)

[OSS1] OKADA, T.-SEKIGUCHI, T.-SHIOTA, Y.: Applications of binomial measures to power sums of digital sums, J. Number Theory 52 (1995), 256-266.

[OSS2] _A generalization of Hata-Yamaguti's results on the Takagi function II: Multinomial case, Japan J. Indust. Appl. Math. 13 (1996), 235-463.

[Pa] PAŠTÉKA, M.: On Four Approaches to Density. VEDA, Bratislava, ISBN 978-80-224-1327-5; Peter Lang, Frankfurt am Main, ISBN 978-3-631-64941-1, 2013.

[Pa1] Remarks on one type of uniform distribution, Unif. Distrib. Theory 2 (2007), no. 1, 79-92.

[P-S] PAŠTÉKA, M.-ČALÁT, T.: Buck's measure density and sets of positive integers containing arithmetic progressions, Math. Slovaca 41 (1991), 283-293.

[P-P] PAŠTÉKA, M.-PORUBSKÝ, V S.: On the distribution of sequences of integers, Math. Slovaca 43 (1993), 521-639.

[P-T] PAŠTÉKA, M.-TICHY, R. F.: Distribution Problems in Dedekind Domains and Submeasures, Annali dell' Universita di Ferrara, Sezione VII-Scienze Matematiche 40 (1994), 191-206. 


\section{LIGIA L. CRISTEA — MILAN PAŠTÉKA}

[S-P] STRAUCH, O-PORUBSKÝ, Š.: Distribution of Sequences: A Sampler. Peter Lang, SAV, Frankfurt am Main, 2005.

[T-W] TICHY, R.F.-WINKLER, R.: Uniform distribution preserving mappings, Acta. Arith. 60 (1991), no. 2, 177-189.

[UP] STRAUCH, O.: Unsolved problems Tatra Mt. Math. Publ. 56 (2013), 109-229, DOI: 10.2478/tmmp-2013-0029. https://math.boku.ac.at/udt/unsolvedproblems.pdf

[W] WEYL, H.: Über die Gleichverteilung von Zahlen mod. Eins, Math. Ann. 77 (1916), 313-352.

Received December 14, 2015

Accepted June 19, 2016

\section{Ligia L. Cristea}

Karl-Franzens-Universität Graz Institut für Mathematik und

Wissenschaftliches Rechnen

Heinrichstrasse 36

8010 Graz

AUSTRIA

E-mail: strublistea@gmail.com

\section{Milan Paštéka}

Trnavska Univerzita,

Pedagogická Fakulta,

Priemyselná 4

P. O. BOX 9

91843 Trnava

SLOVAKIA

E-mail:pasteka@mat.savba.sk 